\title{
L-Carnitine Production Through Biosensor-Guided Construction of the Neurospora crassa Biosynthesis Pathway in Escherichia coli
}

\author{
Pierre Kugler $^{1}$, Marika Trumm ${ }^{1}$, Marcel Frese ${ }^{2}$ and Volker F. Wendisch ${ }^{1 *}$ \\ ${ }^{1}$ Genetics of Prokaryotes, Faculty of Biology and CeBiTec, Bielefeld University, Bielefeld, Germany, ${ }^{2}$ Department \\ of Chemistry, Organic and Bioorganic Chemistry (OCIII), Bielefeld University, Bielefeld, Germany
}

OPEN ACCESS

Edited by:

Yi-Rui Wu,

Shantou University, China

Reviewed by:

Xiaoyuan Wang

Jiangnan University, China Kequan Chen,

Nanjing Tech University, China

${ }^{*}$ Correspondence:

Volker F. Wendisch

volker.wendisch@uni-bielefeld.de

Specialty section:

This article was submitted to Industrial Biotechnology,

a section of the journal

Frontiers in Bioengineering and

Biotechnology

Received: 23 February 2021 Accepted: 26 March 2021

Published: 16 April 2021

Citation:

Kugler P, Trumm M, Frese $M$ and Wendisch VF (2021) L-Carnitine

Production Through

Biosensor-Guided Construction of the Neurospora crassa Biosynthesis

Pathway in Escherichia coli.

Front. Bioeng. Biotechnol. 9:671321. doi: 10.3389/fbioe.2021.671321
L-Carnitine is a bioactive compound derived from L-lysine and S-adenosyl-L-methionine, which is closely associated with the transport of long-chain fatty acids in the intermediary metabolism of eukaryotes and sought after in the pharmaceutical, food, and feed industries. The L-carnitine biosynthesis pathway has not been observed in prokaryotes, and the use of eukaryotic microorganisms as natural L-carnitine producers lacks economic viability due to complex cultivation and low titers. While biotransformation processes based on petrochemical achiral precursors have been described for bacterial hosts, fermentative de novo synthesis has not been established although it holds the potential for a sustainable and economical one-pot process using renewable feedstocks. This study describes the metabolic engineering of Escherichia coli for Lcarnitine production. L-carnitine biosynthesis enzymes from the fungus Neurospora crassa that were functionally active in E. coli were identified and applied individually or in cascades to assemble and optimize a four-step L-carnitine biosynthesis pathway in this host. Pathway performance was monitored by a transcription factor-based L-carnitine biosensor. The engineered $E$. coli strain produced L-carnitine from supplemented L-N $N^{\varepsilon}$ trimethyllysine in a whole cell biotransformation, resulting in $15.9 \mu \mathrm{M}$ carnitine found in the supernatant. Notably, this strain also produced $1.7 \mu \mathrm{M}$ L-carnitine de novo from glycerol and ammonium as carbon and nitrogen sources through endogenous $N^{\varepsilon}$-trimethyllysine. This work provides a proof of concept for the de novo L-carnitine production in E. coli, which does not depend on petrochemical synthesis of achiral precursors, but makes use of renewable feedstocks instead. To the best of our knowledge, this is the first description of L-carnitine de novo synthesis using an engineered bacterium.

Keywords: carnitine, biosynthesis, biosensor, metabolic engineering, biosynthetic cascade, trimethyllysine

\section{INTRODUCTION}

L-Carnitine $[(R)$-3-hydroxy-4-trimethylaminobutyrate $]$ is an essential compound in the intermediary metabolism of eukaryotes, which is involved in the transport of activated longchain fatty acids and products of peroxisomal $\beta$-oxidation into the mitochondria for subsequent completion of $\beta$-oxidation (Vaz and Wanders, 2002). Only the L-isomer is physiologically 
functional in fatty acid transport (Reuter and Evans, 2012), while the other is linked to inhibitory or other toxic effects in different organisms (Rebouche, 1977, 1983; Gross et al., 1986; Li J.M. et al., 2019). In humans, $75 \%$ of the $\mathrm{L}$-carnitine requirement is covered by the diet, while the remaining $25 \%$ are synthesized endogenously (Longo et al., 2016). Carnitine deficiency can lead to a wide range of symptoms that weaken the body (Magoulas and El-Hattab, 2012; Longo et al., 2016). Carnitine can be used as dietary supplement for the treatment of this deficiency (Flanagan et al., 2010; El-Hattab and Scaglia, 2015), but also as feed additive to improve livestock performance (Rehman et al., 2017; Ringseis et al., 2018a,b; Li L. et al., 2019), and as L-carnitine or acyl-L-carnitine esters in further pharmaceutical applications (DiNicolantonio et al., 2013; Chen et al., 2014; Song et al., 2017; Parvanova et al., 2018; Veronese et al., 2018). It is also marketed as food additive and dietary supplement for improving athletic performance and weight management as positive effects on physical performance and weight loss under conditions with disorders have been observed (Fielding et al., 2018), while an improvement in healthy individuals or athletes is debated (Gnoni et al., 2020). The carnitine market of US\$ $\sim 170$ million in 2018 is expected to grow by $4.8 \%$ annually (Grand View Research, 2019; The Insight Partners, 2020).

Racemic D,L-carnitine can be produced chemically from cheap epichlorohydrin and trimethylamine (Kabat et al., 1997), but to obtain enantiomerically pure L-carnitine, either chiral resolution or stereospecific synthesis is required. These approaches are not environmentally friendly, due to the number of reactions, and the need to use chiral starting materials or chiral auxiliaries (Meyer and Robins, 2005; Bernal et al., 2007). Achiral precursors such as crotonobetaine (dehydrated D,L-carnitine), $\gamma$-butyrobetaine, and 3-dehydrocarnitine are converted in highly regio- and enantioselective biotransformations under mild conditions (Bernal et al., 2016). These processes use Pseudomonas sp., Proteus sp., or Escherichia coli (Naidu et al., 2000) and are characterized by a significant reduction in environmental impact, e.g., $89 \%$ less waste to be incinerated, $82 \%$ less waste water, and $76 \%$ less salts (Meyer and Robins, 2005). Fermentative processes hold the potential for de novo biosynthesis from renewable feedstocks and to abandon the petrochemical synthesis of precursors. The use of eukaryotic microorganisms as natural L-carnitine producers suffers from low titers and demanding cultivation conditions with complex media (Wargo and Meadows, 2015; Bernal et al., 2016). Metabolic engineering of established bacterial producers such as E. coli holds the potential to establish de novo L-carnitine production in fermentative one-pot processes.

Biosynthesis of L-carnitine in the filamentous fungus Neurospora crassa initiates with $\mathrm{L}-N^{\varepsilon}$-trimethyllysine (TML) (Horne and Broquist, 1973; Kaufman and Broquist, 1977; Vaz and Wanders, 2002). In eukaryotes, TML is the product of lysosomal or proteasomal degradation of proteins such as calmodulin, myosin, actin, cytochrome c, and histones, which contain $N$-methylated lysine residues from post-translational modification (Strijbis et al., 2010). TML is converted to Lcarnitine in four enzymatic steps (Figure 1), starting with the hydroxylation in $\beta$-position by the TML hydroxylase (TMLH;
EC 1.14.11.8) to yield (2S,3S)-3-hydroxy-TML (HTML) (Sachan and Hoppel, 1980; Vaz et al., 2001; Al Temimi et al., 2016; Reddy et al., 2017). In the next step, HTML is cleaved into glycine and 4-trimethylaminobutyraldehyde (TMABA) by HTML aldolase (HTMLA; EC 4.1.2. "X”). Instead of an aldolase with strict HTML specificity, serine hydroxymethyltransferases (SHMT) (Hulse et al., 1978; Henderson et al., 1982) and threonine aldolases (Strijbis et al., 2009; Franken et al., 2015) show side activities as HTMLA. In the third step, the $\mathrm{NAD}^{+}$-dependent TMABA dehydrogenase (TMABADH; EC 1.2.1.47) oxidizes TMABA to $\gamma$-butyrobetaine $(\gamma$-BB) as shown for Pseudomonas sp. 13CM (Hassan et al., 2008; Bari et al., 2013). Finally, stereoselective hydroxylation of $\gamma$-BB by $\gamma$-BB hydroxylase $(\gamma-\mathrm{BBH}$; EC 1.14.11.1) yields L-carnitine. $\gamma-\mathrm{BBH}$ was not only found in mammals (Lindstedt, 1967; Lindstedt and Lindstedt, 1970), but also in the bacterium Pseudomonas sp. AK 1 (Lindstedt et al., $1967,1970)$, where it is involved in utilization of $\gamma$-BB as the sole source of carbon and nitrogen for growth.

Transfer of the L-carnitine biosynthetic pathway from $N$. crassa to Saccharomyces cerevisiae suffered from low conversion of TML to L-carnitine (Franken et al., 2015). In a similar approach using different genes, production of Lcarnitine from L-lysine in an E. coli whole cell biotransformation was described in a patent application (Kang et al., 2013). Discrepancies between these studies suggest that the $N$. crassa genes coding for $\gamma$-BBH gene and TMABADH have not been unequivocally identified (Kang et al., 2013; Franken et al., 2015), while genes encoding enzymes with TMLH and HTMLA activities are known.

In this study, the enzymes of the L-carnitine biosynthetic pathway from $N$. crassa and their corresponding genes were identified and functionally expressed in E. coli. This organism was chosen as production host because its natural interactions with L-carnitine are already well understood: It uses L-carnitine as compatible solute under hyperosmotic stress conditions and as terminal electron acceptor in anaerobic respiration yielding $\gamma$-BB (Seim et al., 1980; Wargo and Meadows, 2015). Uptake of L-carnitine is mediated by ATP-dependent ABC transport system ProU and to a lesser extent by ProP, a major facilitator superfamily (MFS) proton-metabolite symporter accepting proline, glycine betaine, stachydrine, pipecolic acid, ectoine, taurine, and L-carnitine (Verheul et al., 1998). Anaerobic respiration of L-carnitine involves the enzymes encoded in the divergent operons caiTABCDE and fixABCX (Jung and Kleber, 1991; Eichler et al., 1994; Preusser et al., 1999; Elssner et al., 2001; Jung et al., 2002; Bernal et al., 2007; Bracher et al., 2019; Kugler et al., 2020) that have been used in biotransformation processes of crotonobetaine or D-carnitine to L-carnitine (Castellar et al., 1998, 2001; Obón et al., 1999; Sevilla et al., 2005a,b; Bernal et al., 2007; Arense et al., 2013). We have recently developed a genetically encoded biosensor that responds in a dose-dependent manner to external L-carnitine in a concentration range of $0.1-100 \mu \mathrm{M}$ by expression of the fluorescent reporter mVenus (Kugler et al., 2020). Its use to screen and score enzymes of Lcarnitine biosynthesis in E. coli has been exemplarily shown for betaine:CoA ligases (Kugler et al., 2020). Here, this biosensor was applied to establish the L-carnitine biosynthetic pathway in 

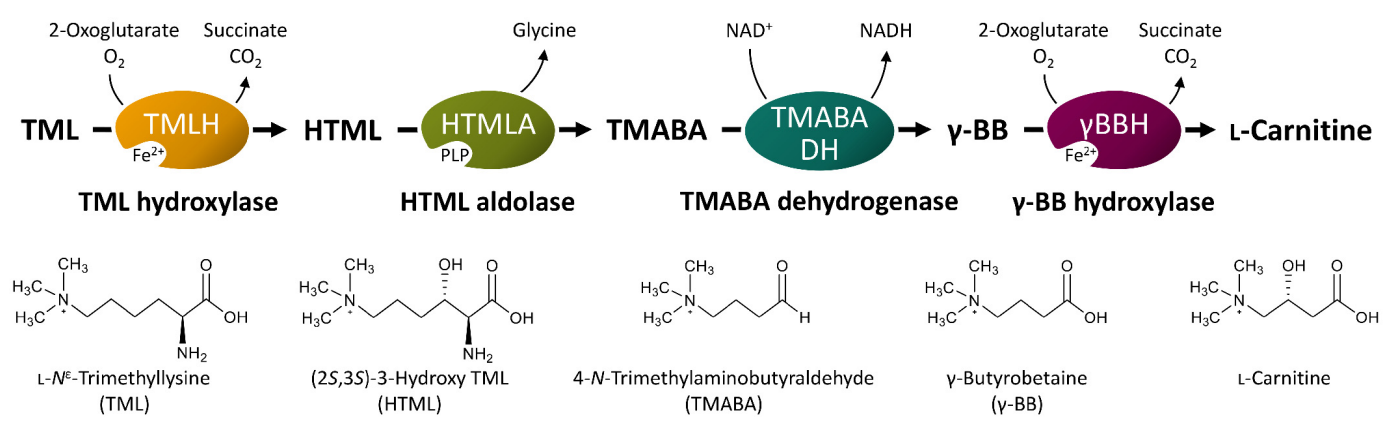

FIGURE 1 | Schematic illustration of L-carnitine synthesis from the precursor TML. PLP, pyridoxal 5'-phosphate; NAD ${ }^{+}$, nicotinamide adenine dinucleotide (oxidized form); NADH, nicotinamide adenine dinucleotide (reduced form). Other abbreviated substances are written in full in the bottom of the figure. Modified from Strijbis et al. (2010).

E. coli. Besides one-pot biotransformation of TML to L-carnitine, L-carnitine could be produced without TML addition. To the best of our knowledge, this is the first fermentative de novo process for L-carnitine production.

\section{MATERIALS AND METHODS}

\section{Bacterial Strains, Recombinant DNA Work, Media, and Growth Conditions}

A complete list of the bacterial strains and plasmids is given in Supplementary Table 2. E. coli DH5 $\alpha$ was used as a cloning host, for plasmid propagation, and as the expression host for the TMABADH enzyme assay and SDS-PAGE. For cloning purposes, cells were grown in lysogeny broth (LB) medium (10 g $\mathrm{L}^{-1}$ tryptone, $5 \mathrm{~g} \mathrm{~L}^{-1}$ yeast extract, and $10 \mathrm{~g} \mathrm{~L}^{-1}$ sodium chloride) at $37^{\circ} \mathrm{C}$ on a shaking incubator (shaking frequency: $180 \mathrm{~min}^{-1}$, eccentricity: $25 \mathrm{~mm}$ ). All media were supplemented with antibiotics where appropriate for selection: kanamycin $\left(50 \mu \mathrm{g} \mathrm{mL}^{-1}\right)$, tetracycline $\left(10 \mu \mathrm{g} \mathrm{mL}^{-1}\right)$, and chloramphenicol $\left(25 \mu \mathrm{g} \mathrm{mL}^{-1}\right)$.

For each tested enzyme of the carnitine biosynthetic pathway from N. crassa, a synthetic gene was designed based on the amino acid sequence. The codon usage in these genes was optimized based on codon usage tables ${ }^{1}$ (Nakamura, 2000) in a codon harmonization tool (Haupka, 2020). The DNA material of these artificial genes was ordered as gene synthesis from BioCat GmbH (Heidelberg, Germany). Artificial optimized 5' untranslated regions ( $5^{\prime}$ UTRs) including the ribosome binding sites (RBS) were generated for each gene and vector combination with the online RBS calculator of the Salis Laboratory of Penn State University ${ }^{2}$ and were introduced via the oligonucleotides during cloning.

The standard molecular genetic techniques were performed as described in detail elsewhere (Green et al., 2012). Genes were amplified by PCR using the respective primers given in Supplementary Table 3 . The oligonucleotides were supplied by Metabion GmbH (Planegg/Steinkirchen, Germany), and the PCR kit containing ALLin HiFi DNA Polymerase was purchased from

${ }^{1}$ www.kazusa.or.jp/codon/

${ }^{2}$ https://salislab.net/software/
highQu GmbH (Kraichtal, Germany) and used according to the manufacturer's instructions. Plasmids were digested using restriction enzymes supplied by Thermo Fisher Scientific Inc. (Waltham, MA, United States) which were used according to the manufacturer's protocols. Insertion of the amplified PCR products was done with Gibson assembly (Gibson et al., 2009). New vectors were confirmed by insert sequencing. The cloning of the vectors is described in detail in the Supplementary Material.

\section{Biosensor Experiments}

The biosensor experiments were conducted as described earlier (Kugler et al., 2020). In short, M9 minimal medium with $24 \mathrm{~g}$ $\mathrm{L}^{-1}$ glycerol as the carbon source and supplemented antibiotics as stated above and $0.1 \mathrm{mM}$ Isopropyl- $\beta$-D-thiogalactopyranosid (IPTG) was used for the cultivation at $37^{\circ} \mathrm{C}$ in 48 -well microtiter FlowerPlates with the Biolector cultivation system (m2p-laboratories GmbH, Baesweiler, Germany) (Funke et al., 2009; Kensy et al., 2009). The medium was inoculated to an optical density at $600 \mathrm{~nm}\left(\mathrm{OD}_{600}\right)$ of 0.1 from precultures that were made in the M9 medium supplemented with $1 \%(\mathrm{v} / \mathrm{v}) \mathrm{LB}$ medium. The formation of biomass was recorded as well as the fluorescence of the yellow fluorescent protein mVenus NB. The fluorescence signal was divided by the biomass signal to obtain the normalized fluorescence signal. The maximum normalized fluorescence signal observed during the whole cultivation was used for the evaluation. Unless stated otherwise, the maximum normalized fluorescence was detected between the middle and end of the exponential growth phase of the culture and for the controls (i.e., empty vector or supplemented water) in the stationary phase.

\section{Assay of TMABA Dehydrogenase Activity in E. coli Crude Extracts}

Escherichia coli DH5 $\alpha$ was used as host for the expression of the potential TMABA dehydrogenases. The enzymes were expressed from the pECXT99A-derived vectors containing the genes TMABADH.1S, TMABADH.1, or TMABADH.2. The cultivation was performed in $500 \mathrm{~mL}$ baffled shake flasks with $50 \mathrm{~mL} \mathrm{LB}$ medium supplemented with antibiotics as stated above on a shaking incubator $\left(37^{\circ} \mathrm{C}\right.$, shaking frequency: $180 \mathrm{~min}^{-1}$, eccentricity: $25 \mathrm{~mm}$ ) with a starting $\mathrm{OD}_{600}$ of 0.1 . At an $\mathrm{OD}_{600}$ 
of 0.6-0.7, expression was induced with $1 \mathrm{mM}$ IPTG and the culture was continued up to an $\mathrm{OD}_{600}$ of $\sim 3.0$ where the cells were harvested at the end of the exponential growth phase by centrifugation $\left(10 \mathrm{~min}, 3,220 \times g, 4^{\circ} \mathrm{C}\right)$. The supernatant was discarded, and the cell pellets were stored at $-80^{\circ} \mathrm{C}$ until further use.

Frozen cell pellets were thawed on ice, resuspended in $1.8 \mathrm{~mL}$ lysis buffer [50 mM potassium phosphate buffer, $1 \mathrm{mM}$ dithiothreitol (DTT); $\mathrm{pH}$ 7.5], and sonicated for $3 \mathrm{~min}$ on ice water (UP200S, Hielscher Ultrasonics GmbH, Teltow, Germany; amplitude $60 \%$, cycle $0.5 \mathrm{~s})$. The cell debris was removed by centrifugation $\left(45 \mathrm{~min}, 28,000 \times \mathrm{g}, 4^{\circ} \mathrm{C}\right.$ ), and the extracts were stored on ice until use in the enzyme assay and for determination of protein concentration with Bradford Reagent (Sigma-Aldrich, St. Louis, MO, United States), using bovine serum albumin as the standard.

TMABA dehydrogenase activity was determined as described before (Bari et al., 2013) with slight modifications. The reaction was followed by measuring the formation of $1 \mathrm{~mol} \mathrm{NADH}$ per consumed mol of TMABA photometrically at $340 \mathrm{~nm}$ $\left(\varepsilon_{N A D H}=6,220 \mathrm{M}^{-1} \mathrm{~cm}^{-1}, \mathrm{~d}=1 \mathrm{~cm}, 30^{\circ} \mathrm{C}\right)$. The reaction mixture of $1 \mathrm{~mL}$ consisted of $150 \mathrm{mM}$ glycine- $\mathrm{NaOH}$ buffer ( $\mathrm{pH}$ 9.5), 2 mM NAD ${ }^{+}, 0.4 \mathrm{mM}$ TMABA iodide, and $250 \mu \mathrm{L}$ crude extract. The basal absorption change was followed for $3 \mathrm{~min}$ and then the reaction was initialized by the addition of TMABA. Where necessary, the crude extract was diluted with the lysis buffer to achieve a rate of absorption change during the measurement in the range of $0.03-0.15$ per min.

\section{Protein Gel Analysis}

The crude extracts from the TMABA dehydrogenase activity assay were further analyzed by sodium dodecyl sulfate polyacrylamide gel electrophoresis (SDS-PAGE) as described elsewhere (Green et al., 2012) using the Mini-PROTEAN Tetra Cell \#1658000EDU System from Bio-Rad Laboratories Inc. (Hercules, CA, United States). The amount of protein used per sample was $12 \mu \mathrm{g}$, and the PageRuler Prestained Protein Ladder 10-180 kDa (Thermo Fisher Scientific Inc., Waltham, MA, United States) was used as molecular weight marker.

\section{Carnitine Production Experiment With Recombinant E. coli Expressing the Carnitine Biosynthetic Pathway From N. crassa}

The cultivation was done in $100 \mathrm{~mL}$ baffled shake flasks with $10 \mathrm{~mL}$ modified M9 medium with $30 \mathrm{~g} \mathrm{~L}^{-1}$ glycerol as carbon source and with antibiotics as described above. Nitrogen is the limiting factor for biomass formation in the composition of the M9 medium, which is why $\mathrm{NH}_{4} \mathrm{Cl}$ was supplemented in this experiment to double the concentration to $2 \mathrm{~g} \mathrm{~L}^{-1}$ to reach a higher biomass in this cultivation. The cultures were inoculated to an $\mathrm{OD}_{600}$ of 0.1 from precultures that were made in standard M9 medium supplemented with $1 \%(\mathrm{v} / \mathrm{v}) \mathrm{LB}$ medium and placed in a shaking incubator $\left(37^{\circ} \mathrm{C}, 180 \mathrm{~min}^{-1}\right.$ shaking frequency, $25 \mathrm{~mm}$ eccentricity). At an $\mathrm{OD}_{600}$ of $0.5-0.6$, expression was induced with $0.1 \mathrm{mM}$ IPTG and the substrate TML was added at a concentration of $1 \mathrm{mM}$. Cultivation was continued for $48 \mathrm{~h}$, and then a $2 \mathrm{~mL}$ sample was drawn from which the cell pellet and supernatant were obtained by centrifugation $(10 \mathrm{~min}, 12,000 \times g$, $\left.4^{\circ} \mathrm{C}\right)$. After washing the cells with saline $\left(9 \mathrm{~g} \mathrm{~L}^{-1} \mathrm{NaCl}\right)$, the cells were pelleted again by centrifugation $(10 \mathrm{~min}, 12,000 \times g$, $4^{\circ} \mathrm{C}$ ) and then all samples were frozen at $-20^{\circ} \mathrm{C}$ until analysis via LC-MS.

\section{LC-MS Measurement}

The supernatant samples were thawed on ice and then centrifuged $\left(10 \mathrm{~min}, 12,000 \times g, 4^{\circ} \mathrm{C}\right)$ to separate possible precipitates from freezing. Subsequently, the undiluted samples were analyzed by LC-MS. The cell pellets were resuspended in $800 \mu \mathrm{L}$ of ice-cold $80 \%(\mathrm{v} / \mathrm{v})$ methanol, mixed with $0.35 \mathrm{~g}$ of $0.2 \mathrm{~mm}$ zirconia-silica beads (BioSpec Products Inc., Bartlesville, $\mathrm{OK}$, United States) in $1.5 \mathrm{~mL}$ microtubes, and cooled on ice. The suspensions were placed in the Digital Disruptor Genie Cell Disruptor (Scientific Industries Inc., Bohemia, NY, United States), and the cells were disrupted for $3 \mathrm{~min}$ at full speed. Next, cell debris and beads were separated by centrifugation (10 min, $12,000 \times g, 4^{\circ} \mathrm{C}$ ). The methanol was evaporated from $600 \mu \mathrm{L}$ of the resulting supernatant in a vacuum concentrator (Eppendorf AG, Hamburg, Germany) for $4 \mathrm{~h}$ at $45^{\circ} \mathrm{C}$. The residue was re-dissolved in $120 \mu \mathrm{L}$ water and analyzed by LCMS. For the LC-MS measurement, an Agilent 6220 TOF-MS with a Dual ESI-source and a 1200 HPLC system (Agilent Technologies, Inc., Santa Clara, CA, United States) with a Hypersil Gold C18 column $(1.9 \mu \mathrm{m}, 50 \times 2.1 \mathrm{~mm}$; Thermo Fisher Scientific Inc., Waltham, MA, United States) was used. Solvent A consisted of $94.9 \%(\mathrm{v} / \mathrm{v})$ water, 5\% (v/v) acetonitrile, and $0.1 \%(\mathrm{v} / \mathrm{v})$ formic acid, and solvent $\mathrm{B}$ was composed of $5 \%(\mathrm{v} / \mathrm{v})$ water, $94.9 \%(\mathrm{v} / \mathrm{v})$ acetonitrile, and $0.1 \%(\mathrm{v} / \mathrm{v})$ formic acid. A gradient was started at $0 \% \mathrm{~B}$ when $5 \mu \mathrm{L}$ sample was injected and increased to $98 \% \mathrm{~B}$ over $11 \mathrm{~min}$, went back to $0 \% \mathrm{~B}$ in $0.5 \mathrm{~min}$, which was held for $3.5 \mathrm{~min}$ to a total run time of $15 \mathrm{~min}$. The flow rate was $300 \mu \mathrm{L} \mathrm{min}-1$, and column oven temperature was at $40^{\circ} \mathrm{C}$. The extended dynamic range mode was used with a Dual-ESI source, operating with a spray voltage of $2.5 \mathrm{kV}$. The data were analyzed with MassHunter Workstation Software Version B.07.00 (Agilent Technologies, Inc., Santa Clara, CA, United States). Carnitine was identified in an accurate mass measurement from its mass to charge ratio $(\mathrm{m} / \mathrm{z}$ ) of 162.11267 (deviation $<5 \mathrm{ppm}$ to calculated $\mathrm{m} / \mathrm{z}$ of 162.11247). The mass spectrum of L-carnitine is shown in Supplementary Figure 2. The quantitative analysis was performed using a set of L-carnitine standard samples with the concentrations (in $\mu \mathrm{M}$ ): $0,1.56,3.136 .25,12.5,25$, and 50. From the resulting total ion count (TIC), a regression line was calculated $\left(R^{2}=0.998\right)$, which was used as the calibration curve. Intracellular carnitine concentrations were calculated from the carnitine concentration determined for the cell pellet extract and the total cell volume of the cell pellet, which was calculated from the final $\mathrm{OD}_{600}$ of the $2 \mathrm{~mL}$ samples at $48 \mathrm{~h}$ fermentation and the OD specific total cell volume for starved E. coli cells in the stationary phase of $3.3 \mu \mathrm{L} \mathrm{mL}^{-1} \mathrm{OD}_{600}{ }^{-1}$ (Volkmer and Heinemann, 2011). 


\section{Analytical Quantification of Glycerol in the Carnitine Production Experiment}

Samples were drawn at $24 \mathrm{~h}$ and $48 \mathrm{~h}$ cultivation time. The supernatant was collected by centrifugation $(10 \mathrm{~min}, 12,000 \times \mathrm{g}$, $4^{\circ} \mathrm{C}$ ) and frozen at $-20^{\circ} \mathrm{C}$ until analysis via HPLC. For analysis, the samples were thawed on ice and then centrifuged $(10 \mathrm{~min}$, $12,000 \times g, 4^{\circ} \mathrm{C}$ ) to separate possible precipitates from freezing. Subsequently, the undiluted samples were analyzed on an HPLC system (1200 series, Agilent Technologies Deutschland $\mathrm{GmbH}$, Böblingen, Germany) with an organic acid resin column $(300 \mathrm{~mm} \times 8 \mathrm{~mm})$ and the corresponding precolumn $(40 \mathrm{~mm} \times 8 \mathrm{~mm}$ ) (Chromatographie-Service $\mathrm{GmbH}$, Langerwehe, Germany) heated to $60^{\circ} \mathrm{C}$. The mobile phase was $5 \mathrm{mM}$ sulfuric acid in water (Milli-Q grade) with an isocratic flow rate of $0.8 \mathrm{~mL} \mathrm{~min}{ }^{-1}$. A refractive index detector was used for molecule detection. A calibration curve $\left(R^{2}=0.999\right)$ was generated from glycerol standards with the concentrations (in $\mathrm{g}$ $\left.\mathrm{L}^{-1}\right): 1,2,4,8,16$, and 32 .

\section{Reagents}

Unless specified, all chemical standard reagents were purchased in the highest grade available from Sigma-Aldrich (St. Louis, MO, United States) and Carl Roth GmbH + Co., KG (Karlsruhe, Germany). L- $N^{\varepsilon}$-trimethyllysine was purchased from Glentham Life Sciences Ltd. (Corsham, United Kingdom), and L-carnitine and $\gamma$-butyrobetaine were ordered at Sigma-Aldrich (St. Louis, MO, United States). The TMABA iodide was prepared from 4aminobutyraldehyde diethyl acetal (Acros Organics B.V.B.A, Fair Lawn, NJ, United States) as described in the Supplementary Material.

\section{RESULTS}

\section{Selection and Cloning of the L-Carnitine Biosynthetic Pathway Genes From Neurospora crassa}

The selection of the potential enzymes for the L-carnitine biosynthetic pathway in this study was based on two sets of $N$. crassa genes that were previously considered for a transfer of the pathway to heterologous hosts (Kang et al., 2013; Franken et al., 2015). An overview of the enzymes and their respective genes used in this study is given for each step in Supplementary Table 1. Both sets share the enzymes for the first two steps (TMLH and HTMLA). The TMLH has been previously cloned and confirmed in S. cerevisiae (Swiegers et al., 2002), and for the HTMLA, the gene of the N. crassa SHMT was selected, as it additionally possesses aldolase activity (Kruschwitz et al., 1993; Franken et al., 2015). In case of the TMABADH, a third variant (TMABADH.1) was tested here in addition to the versions TMABADH.1S (Kang et al., 2013) and TMABADH.2 (Franken et al., 2015). Both TMABADH.1S and TMABADH.1 are based on the ORF NCU00378; however, TMABADH.1 (549 AA; RefSeq: XP_957264.2) is longer than TMABADH.1S (495 AA; XP_957264.1), which results from a reannotation extending the ORF to a start codon 153 bp upstream. For $\gamma$-BBH, two different enzymes termed $\gamma$ BBH.1 (Kang et al., 2013) and $\gamma$ BBH.2 (Franken et al., 2015) were selected from the literature and chosen for this study.

A codon-harmonized gene and corresponding optimized 5' UTRs including the RBS were designed for each variant using a codon harmonization tool (Haupka, 2020) and codon usage tables $^{3}$ (Nakamura, 2000), and the online RBS calculator of the Salis Laboratory of Penn State University (see text footnote 1).

\section{Screening for Functional $\gamma$-Butyrobetaine Hydroxylases}

For the screening of the $\gamma$-butyrobetaine hydroxylases, the genes $\gamma$ BBH.1 and $\gamma$ BBH.2 (Supplementary Table 1) were cloned into the expression plasmid pECXT99A. To score $\gamma$-butyrobetaine hydroxylase activity, an L-carnitine biosensor strain was used, and fluorescence was measured upon extracellular addition of $\gamma$-BB. Specifically, the enzymes were tested for L-carnitine formation from $\gamma$-BB in the L-carnitine biosensor strain E. coli BW25113 $\Delta$ cai-fix (pGP2-Sensor1-caiCD) (see Supplementary Table 2), which was transformed with pECXT99A- $\gamma$ BBH.1, pECXT99A- $\gamma$ BBH.2, or pECXT99A as empty vector control. A microbioreactor cultivation was performed using the Biolector system (m2p-laboratories GmbH, Baesweiler, Germany) (Funke et al., 2009; Kensy et al., 2009) with the resulting strains in M9 minimal medium supplemented with $100 \mu \mathrm{M} \gamma$-BB or water as control and the maximum normalized mVenus fluorescence was measured (Figure 2). M9 minimal medium was selected for all cultivation experiments because it significantly increases the utility and sensitivity of the biosensor used (Kugler et al., 2020).

As expected, no L-carnitine biosensor fluorescence signal was measured in the empty vector control by the addition of $\gamma$-BB. While $\gamma$ BBH.1 showed no activity when $\gamma$-BB was supplemented, an L-carnitine biosensor fluorescence signal could be detected for $\gamma$ BBH.2 upon addition of $\gamma$-BB. Thus, $\gamma$ BBH.2 was confirmed in this screening as functionally active $\gamma$-butyrobetaine hydroxylase and used for further work on the L-carnitine biosynthetic pathway.

\section{Screening for Functional TMABA Dehydrogenases}

An in vitro photometric enzyme assay based on cell extracts was chosen for screening TMABA dehydrogenase activity, as it is easily feasible due to the co-conversion of $\mathrm{NAD}^{+}$to $\mathrm{NADH}$ in the TMABADH reaction (Figure 3). A screening with the biosensor as for the $\gamma$-BBH was not chosen because external supplementation of aldehydes can have toxic effects on the cells due to their high reactivity and it is uncertain that the aldehyde is taken up by the cells (Kunjapur and Prather, 2015). E. coli DH5 $\alpha$ was transformed with pECXT99A-based plasmids harboring the dehydrogenases TMABADH.1S, TMABDH.1, or TMABADH.2 (Supplementary Table 1) and with pECXT99A as the empty vector control. Cell extracts were prepared from the induced strains and examined in an enzyme assay in which the reaction was photometrically monitored by measuring the formation of $\mathrm{NADH}$ (Figure 3).

${ }^{3}$ www.kazusa.or.jp/codon/ 


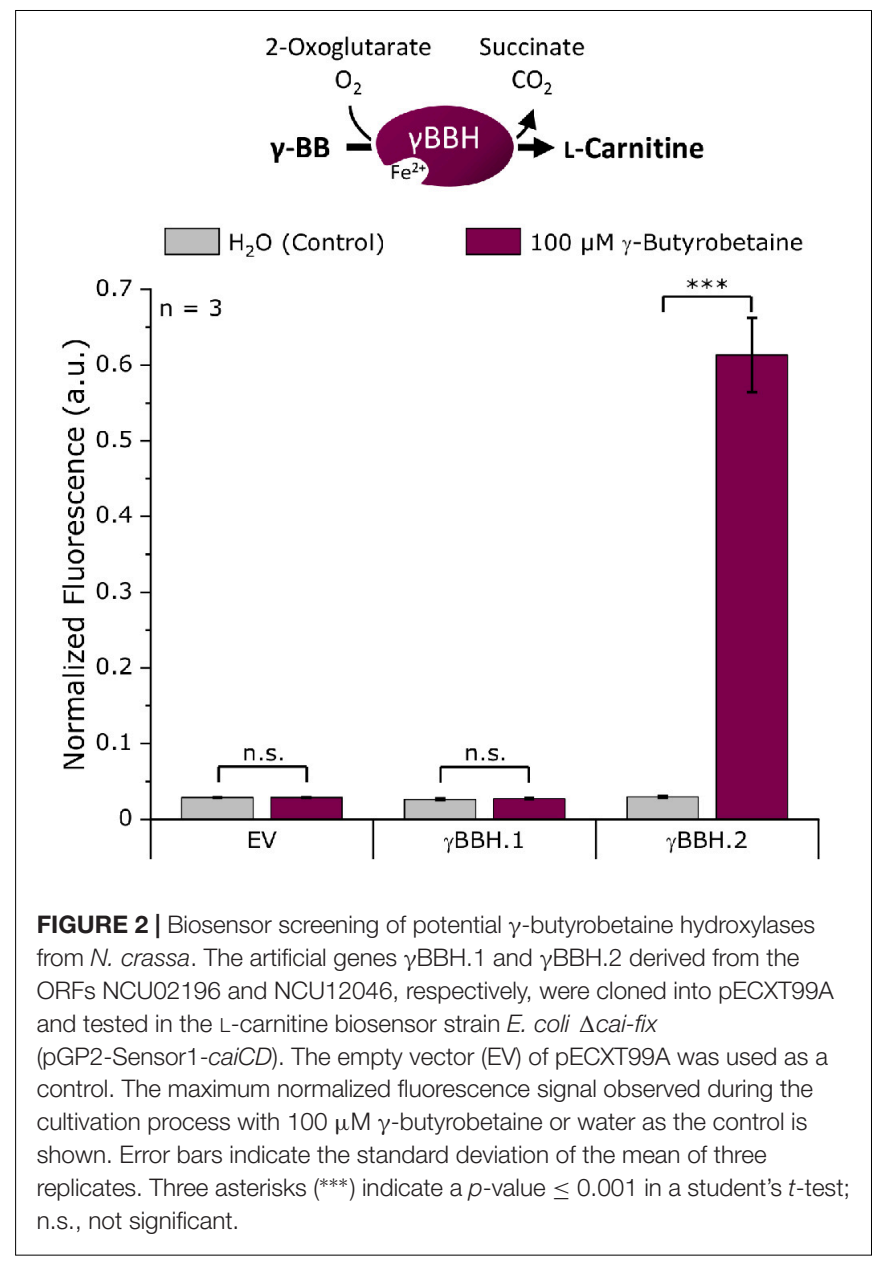

As expected, no activity could be detected in the empty vector control, and neither TMABADH.1 nor TMABADH.2 showed activity. By contrast, TMABA dehydrogenase activity was revealed for TMABADH.1S (Figure 3). Since TMABADH.1 and TMABADH.1S share a great similarity on the amino acid level, it was speculated whether the expression of TMABADH.1 was affected by the N-terminal modification compared to TMABADH.1S. To investigate this, an SDS-PAGE was performed from the same cell extracts. Indeed, protein abundance of TMABADH.1 was significantly lower as compared to TMABADH.1S and TMABADH.2 (Supplementary Figure 1). Since TMABADH.1S could be expressed well and showed about $0.9 \mathrm{U} \mathrm{mg}^{-1}$ activity, it was selected for further work on the carnitine biosynthetic pathway.

\section{Assembly of the L-Carnitine Biosynthetic Pathway}

After efficient enzymes for the last and penultimate step of the L-carnitine biosynthetic pathway had been identified, enzymes for the first two steps were selected: TML hydroxylase and an HTML aldolase (Supplementary Table 1). With the biosensor as a read-out tool for L-carnitine formation, the pathway was gradually assembled in the biosensor strain and the formation

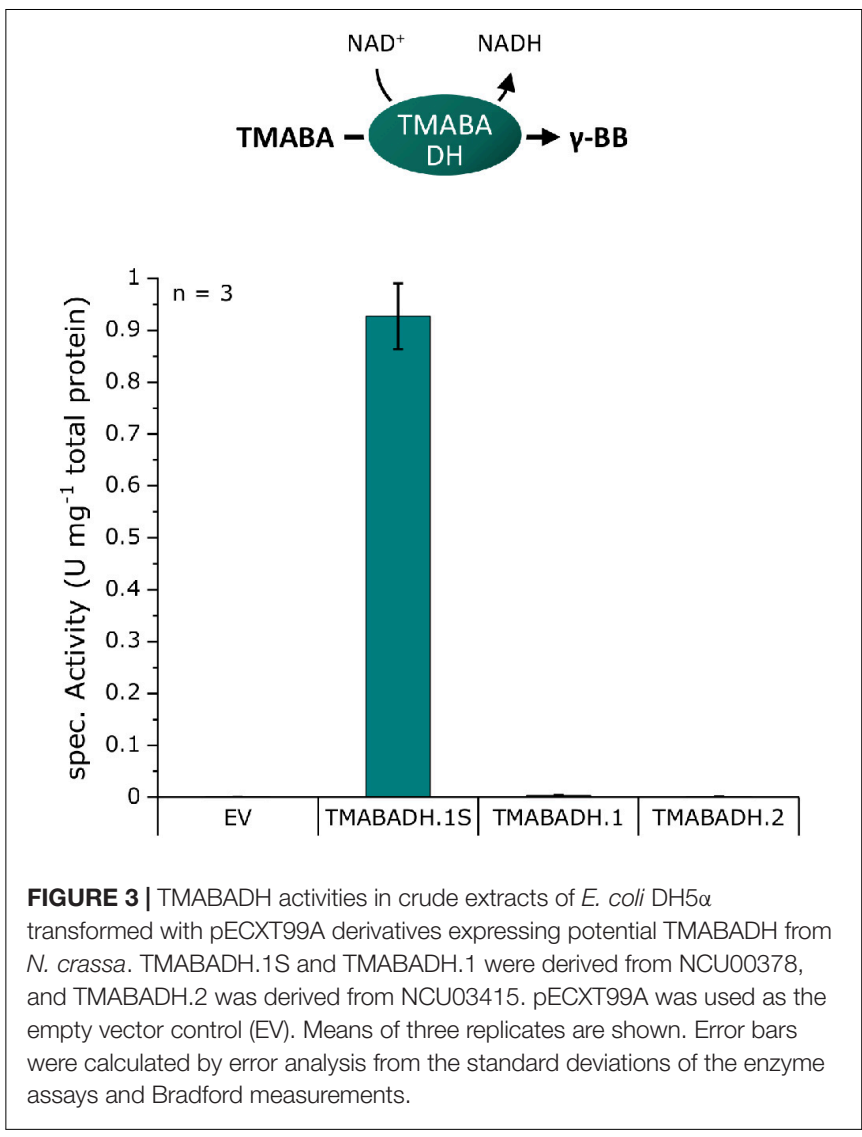

of L-carnitine from externally supplemented TML was studied. Additionally, this experimental setup was used to investigate the TMABADH variants in vivo in combination with the other pathway enzymes. The L-carnitine biosensor strain E. coli BW25113 $\Delta$ cai-fix (pGP2-Sensor1-caiCD) was transformed with different plasmid combinations to assemble the full pathway. The TMABADH variants were included to validate the results from the in vitro assay in an in vivo biolector cultivation experiment. M9 minimal medium supplemented with $100 \mu \mathrm{M}$ TML or water as control was used and the maximum normalized mVenus fluorescence was measured (Figure 4). Individual expression of $\gamma \mathrm{BBH} .2$ or TMLH (combinations A and B, respectively) did not result in an L-carnitine biosensor signal upon addition of TML as precursor. Cascading of all four enzymes in vivo gave a strong $\mathrm{L}$-carnitine biosensor signal (combination $\mathrm{H}$ ) indicating that the pathway operated as anticipated.

Surprisingly, cascades with TMLH, but lacking one or more (active) enzymes (combination C, D, E, F, and G) also showed L-carnitine biosensor signal output, albeit it was significantly lower than that of the complete cascade (combination $\mathrm{H}$ ). This suggests that enzymes present in the E. coli host strain are active in the conversion of HTML to $\gamma$-BB. However, currently their identities remain unknown. For further work on the pathway, the cascade TML hydroxylase/HTML aldolase/TMABADH.1S/ $\gamma$ BBH.2 (combination $\mathrm{H}$ ) was chosen. 


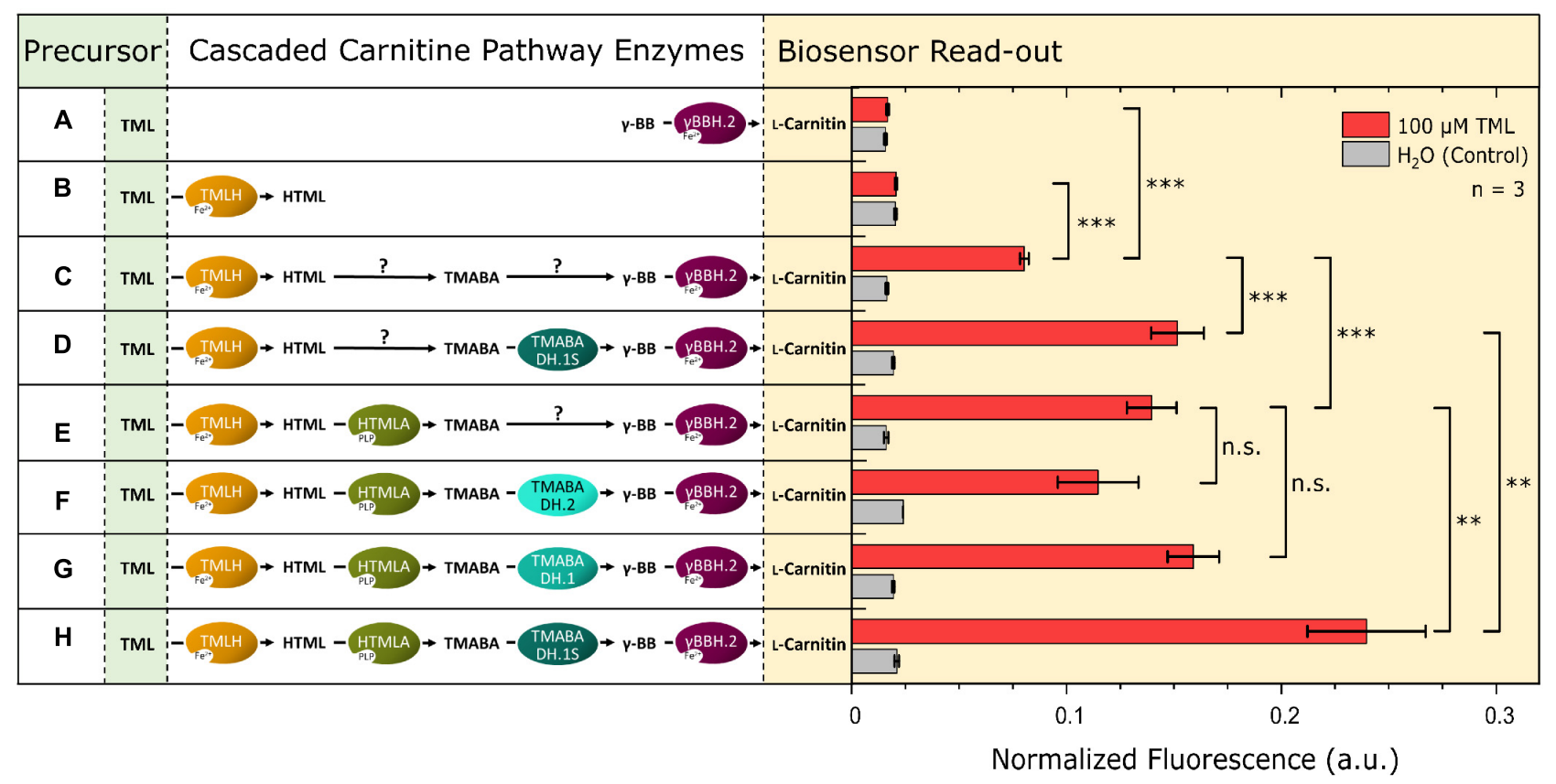

FIGURE 4 | Assembly of the four steps of the L-carnitine biosynthetic pathway of $N$. crassa in $E$. coli. The expression of a gene encoding a cascaded enzyme is depicted by an oval on the left side. Arrows marked with a "?" indicate that the reaction may proceed via endogenous enzymes although no dedicated gene was overexpressed. Plasmid pPLib3 was used for the individual expression of TMLH (pPLib3-TMLH; Combinations B, C, and D) and together with HTMLA as synthetic operon (pPLib3-TMLH-HTMLA; Combinations E, F, G, and H) and as empty vector control (pPLib3; Combination A). pECXT99A was used to express $\gamma$ BBH.2 individually (pECXT99A- $\gamma$ BBH.2; Combinations A, C, and E) or in a synthetic operon with one of the TMABADH variants (pPLib3-TMABADH.1S- $\gamma$ BBH.2; Combinations D and H, pPLib3-TMABADH.1- $\gamma$ BBH.2; Combination G, or pPLib3-TMABADH.2- $\gamma$ BBH.2; Combination F) and as empty vector control (Combination B). The shown enzyme combinations were tested in the L-carnitine biosensor strain E. coli $\Delta$ cai-fix (pGP2-Sensor1-caiCD). The maximum normalized fluorescence signal observed during the cultivation process with $100 \mu \mathrm{M} \mathrm{TML}$ (red columns) or water (gray columns) as the control is shown. Error bars indicate the standard deviation of the mean of three replicates. Three asterisks $\left(^{* * *}\right)$ indicate a $p$-value $\leq 0.001$ in a student's $t$-test, ${ }^{* *} p$-value $\leq 0.01$, and n.s., not significant.

\section{Improving the L-Carnitine Biosynthesis Pathway for de novo Production by \\ E. coli}

After having selected the enzymes for the L-carnitine biosynthetic pathway, expression of the four genes was optimized by combining the individual genes in a single plasmid as a synthetic operon. The plasmid pTrc99A was used as backbone and the previously identified genes for the pathway (TMLH, HTMLA, TMABADH.1S, and $\gamma$ BBH.2; Figure 4, combination H) were cloned with optimized $5^{\prime}$ UTRs in a single synthetic operon. The gene order was the same as that of the reaction steps, and the constructed plasmid for conversion of TML to L-carnitine was called pTrc99A-TML2Car. The plasmid was transformed in the L-carnitine biosensor strain E. coli BW25113 $\triangle$ cai-fix (pGP2-Sensor1-caiCD) and tested in parallel with pTrc99A as empty vector control in a biolector experiment. The medium was supplemented with either $100 \mu \mathrm{M}$ TML or water as control, and the normalized mVenus fluorescence was monitored over the cultivation time (Figure 5A). In the strain harboring the L-carnitine biosynthetic pathway (labeled TML2Car), the supplementation of $100 \mu \mathrm{M}$ TML resulted in a fluorescence response of the L-carnitine biosensor. The maximum signal (Figure 5B) was more than twofold higher than the signal previously measured for the pathway when its genes were expressed from two separate plasmids (Figure 4, Combination H). Interestingly, strain TML2Car showed a significantly increased biosensor signal compared to the empty vector strain even when no TML was supplemented (Figure 5B, $\mathrm{H}_{2} \mathrm{O}$ control). This may indicate de novo Lcarnitine formation from endogenous TML. In contrast to the measurement with supplemented TML, the maximum normalized fluorescence was detected in the stationary phase and not at the end of the exponential growth phase (Figure 5A). The origin of TML in the E. coli host is unknown. While interesting from a physiological point of view, it was not required for the L-carnitine biosynthetic cascade developed here.

\section{LC-MS Analysis of L-Carnitine Production by Recombinant E. coli Expressing the L-Carnitine Biosynthetic Pathway from $N$. crassa}

The heterologous expression of the genes for the full Lcarnitine biosynthesis cascade by the plasmid pTrc99A-TML2Car revealed L-carnitine production not only from the supplemented precursor TML, but also without it, providing evidence of de novo L-carnitine biosynthesis (Figure 5). To further substantiate these results, L-carnitine formation was studied in a shake flask 

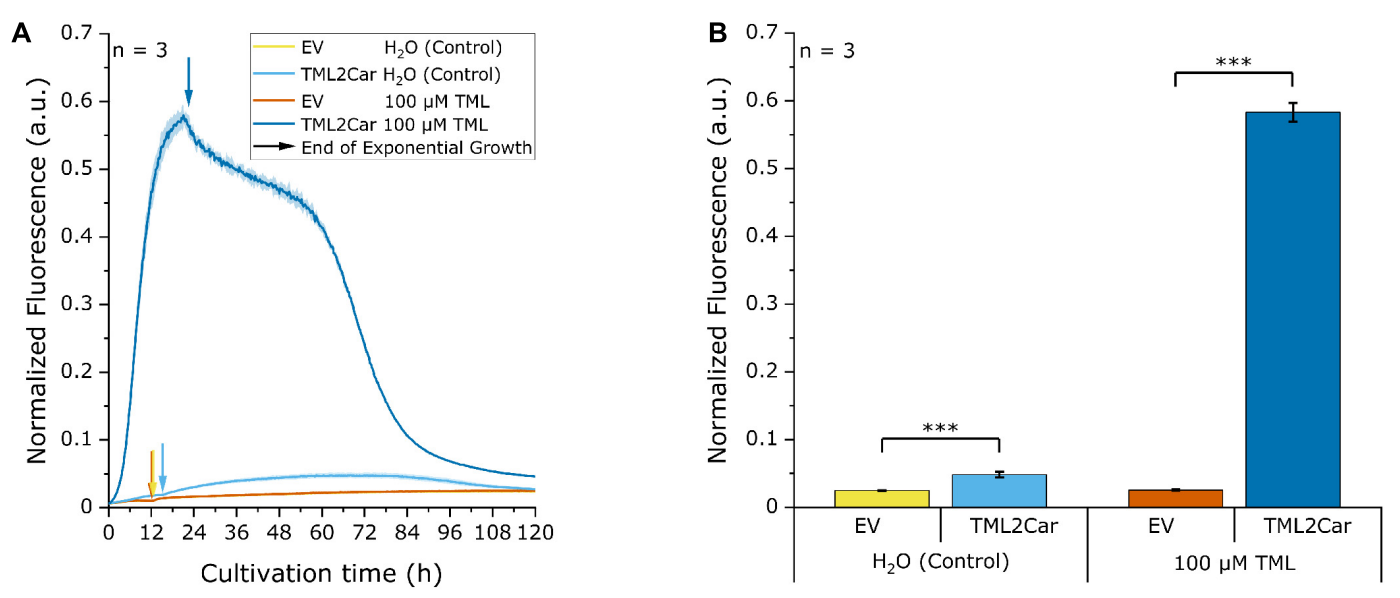

FIGURE 5 | Biosensor experiment with the synthetic L-carnitine biosynthesis pathway operon. The pathway genes were cloned in the order of the respective reaction steps into pECXT99A resulting in plasmid pECXT99A-TML2Car and tested in the L-carnitine biosensor strain E. coli $\triangle$ cai-fix (pGP2-Sensor1-caiCD). The empty vector (EV) of pTrc99A was used as a control. In the cultivation, either $100 \mu \mathrm{M}$ TML was supplemented, or water was used as control. (A) Development of the normalized fluorescence signal over the cultivation process. The yellow (EV, $\mathrm{H}_{2} \mathrm{O}$ ) and orange lines (EV, $\left.100 \mu \mathrm{M} \mathrm{TML}\right)$ are largely overlapping. The end of the exponential growth phase and transition to the stationary growth phase is marked with arrows for each condition. (B) Analogous to the previous readouts, the maximum normalized fluorescence signal for each cultivation was extracted from (A) and analyzed for significance of values between TML2Car and the respective EV control. Error bars indicate the standard deviation of the mean of three replicates. Three asterisks $(* * *)$ indicate a $p$-value $\leq 0.001$ in a student's $t$-test.

production experiment, and extracellular and intracellular Lcarnitine concentrations were analyzed by LC-MS. The strains were similar to those used in the previous experiment but lacked the biosensor plasmid. E. coli strains BW25113 $\Delta$ caifix (pTrc99A-TML2Car) and empty vector control BW25113 $\Delta$ cai-fix (pTrc99A) were cultivated for $48 \mathrm{~h}$ in modified M9 minimal medium with increased levels of glycerol and ammonium as carbon and nitrogen sources and with and without supplementation of $1 \mathrm{mM}$ TML. LB or another complex medium was not used to keep the cultivation comparable to the biosensor experiments and to avoid potential TML and carnitine sources in the medium, e.g., yeast extract. The growth and carbon source consumption were followed over the cultivation time (Figure 6A). The EV strain grew slightly faster with a maximum growth rate of $0.52 \mathrm{~h}^{-1}$ and an average final $\mathrm{OD}_{600}$ of 17.3, whereas the TML2Car strain grew at a rate of $0.46 \mathrm{~h}^{-1}$ to an average $\mathrm{OD}_{600}$ of 15.3 at the end of the fermentation after $48 \mathrm{~h}$, with all strains converting $\sim 50 \%$ of the provided carbon source glycerol.

The supernatant and cells pellets were analyzed by LC-MS, and the extracellular and intracellular L-carnitine concentrations were determined (Figure 6B). The mass of L-carnitine $(\mathrm{m} / \mathrm{z}$ ratio 162.11247 ) could be scored in the supernatants and cells of the strain expressing the L-carnitine biosynthetic pathway (labeled TML2Car), but not in the empty vector control strain. An extracellular concentration of $1.7 \mu \mathrm{M}$ was produced without supplementation of TML. When $1 \mathrm{mM}$ of the precursor was supplemented, $15.9 \mu \mathrm{M}$ L-carnitine could be found in the supernatant $(1.6 \%$ molar yield as judged by the L-carnitine and TML concentrations in the culture medium). The intracellular L-carnitine concentrations were similar to the supernatants but slightly higher.

\section{DISCUSSION}

Implementing a cascade of enzymes from $N$. crassa into E. coli enabled biotransformation of TML to L-carnitine as demonstrated using a genetically encoded L-carnitine biosensor and by LC-MS analysis. Moreover, the metabolically engineered E. coli strain enabled de novo production of L-carnitine from a mineral salts medium with glycerol and ammonium as carbon and nitrogen sources.

The ability of $E$. coli to synthesize sufficient TML for de novo production of L-carnitine came as a surprise since E. coli synthesizes TML to a much lesser extent than eukaryotic cells (Klagsbrun and Furano, 1975; Zhang et al., 2018). Methyltransferase PrmA methylates two lysyl residues of protein L11 from the large ribosomal subunit (Colson et al., 1979; Vanet et al., 1994) and free TML accumulates upon degradation of methylated protein L11 similar to TML release in eukaryotes (Vaz and Wanders, 2002). Our observation of accumulation of L-carnitine in the stationary phase is consistent with release of TML by protein degradation, which primarily occurs in this growth phase. Alternatively, it is possible that PrmA methylates free lysine to free TML as a side reaction as demonstrated for the RuBisCo large subunit $N$-methyltransferase (RBCMT) from Pisum sativum (Trievel et al., 2003).

Endogenous enzymes of E. coli complemented the fourstep pathway when only TMLH and $\gamma-\mathrm{BBH}$ were provided (Figure 4; Combination $\mathrm{C}$ ), thus, enzymes with (side) activities as HTML aldolase and TMABA dehydrogenase exist in E. coli. The low-specificity L-threonine aldolase LtaE, which cleaves several L-3-hydroxy- $\alpha$-amino acids, like HTML, to glycine and a corresponding aldehyde and which may accommodate molecules larger than L-threonine in its large substrate binding pocket (di Salvo et al., 2014), may exhibit side activity as HTML 

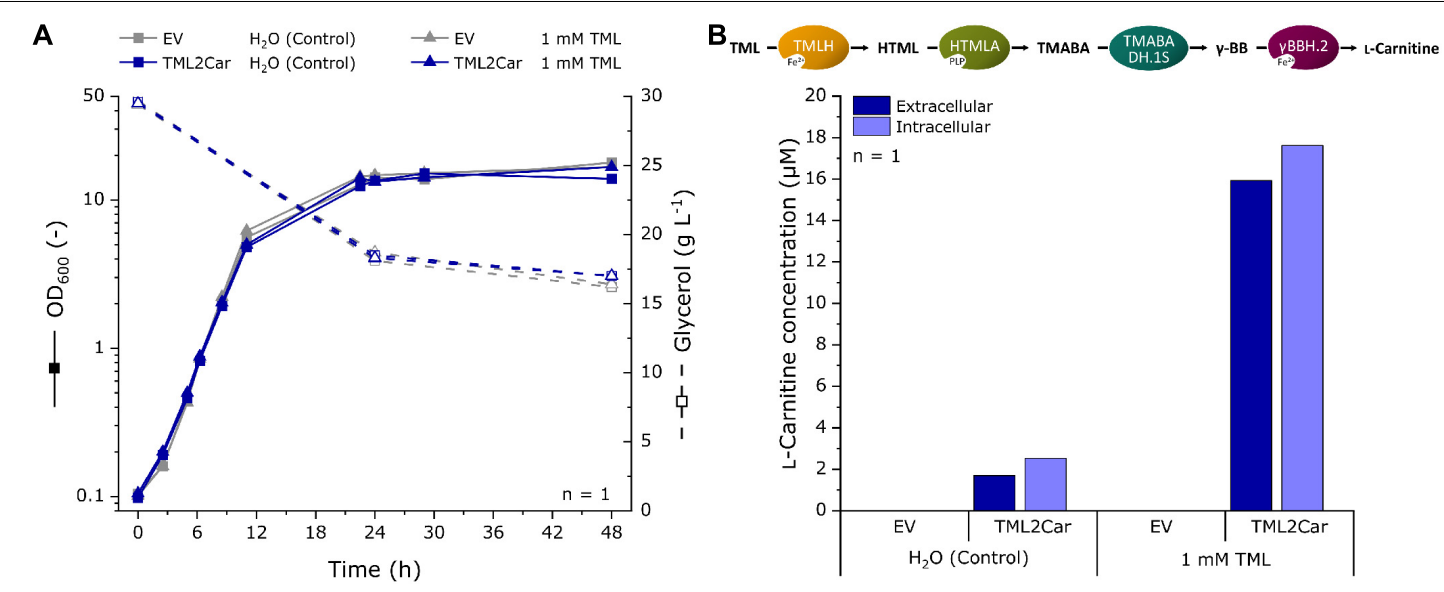

FIGURE 6 | Fermentation for L-carnitine production in modified M9 minimal medium with supplementation of $1 \mathrm{mM}$ TML or water as a control using recombinant E. coli expressing the L-carnitine biosynthetic pathway from N. crassa. The pathway was expressed in E. coli BW25113 $\triangle$ cai-fix from plasmid pTrc99A-TML2Car. The empty vector (EV) pTrc99A was used as control. (A) Growth curve and residual glycerol concentrations of the fermentation experiment. (B) LC-MS analysis of the extracellular and intracellular L-carnitine concentration at the end of the cultivation after $48 \mathrm{~h}$. $\mathrm{L}$-carnitine was identified by its mass to charge ratio ( $\mathrm{m} / \mathrm{z}$ ) of 162.11247. The detection limit was $0.5 \mu \mathrm{M}$. Intracellular concentrations were determined from the L-carnitine concentration in cell pellet extracts and the calculated total cell volume of the pellet.

aldolase. Alternatively, this activity may be catalyzed by E. coli's SHMT GlyA, which shares $48 \%$ amino acid identity with the $N$. crassa SHMT that was used in this study (Schirch et al., 1985). In the case of TMABA dehydrogenase, numerous aldehyde dehydrogenases native to $E$. coli may possess this side activity (Sophos and Vasiliou, 2003). The most likely candidates among them may be $\gamma$-aminobutyraldehyde dehydrogenase PatD that is active with unmethylated TMABA ( $\gamma$-aminobutyraldehyde) or with betaine aldehyde (Gruez et al., 2004; Samsonova et al., 2005) and PuuC, a non-specific aldehyde dehydrogenase that oxidizes all aldehydes in putrescine catabolism including $\gamma$-aminobutyraldehyde (Schneider and Reitzer, 2012). It remains to be shown if overexpression of the genes for these enzymes is beneficial for de novo production of $\mathrm{L}$-carnitine since accumulation of intermediates in the conversion of TML to L-carnitine was not observed.

The E. coli strain constructed here produced $1.7 \mu \mathrm{M}$ L-carnitine de novo, which is fourfold higher than by biotransformation from $100 \mu \mathrm{M}$ supplemented TML by recombinant S. cerevisiae (Franken et al., 2015). However, our finding that addition of $1 \mathrm{mM}$ TML to the $E$. coli strain constructed here improved L-carnitine production about 10 -fold (1.7 $\mu \mathrm{M}$ as compared to around $16 \mu \mathrm{M}$ ) suggested that the supply of the precursor TML may limit de novo production. It remains to be shown if overexpression of endogenous prmA or heterologous expression of the RBCMT gene from $P$. sativum increases de novo production of $\mathrm{L}$-carnitine by the $E$. coli strain constructed here. Alternatively, a bona fide L-lysine methyltransferase (K-NMT) yielding TML may be used. Such an enzyme has been reported for $N$. crassa (Borum and Broquist, 1977). The gene NCU03826 that was speculated to encode K-NMT in a patent application (Kang et al., 2013) did not result in a biosensor response that would indicate improved L-carnitine levels and the supplementation of lysine and methionine as precursors did not change this outcome (data not shown). Consistent with this finding, NCU03826 did not function as K-NMT in the S. cerevisiae strain (Franken et al., 2015). Thus, the gene for a bona fide K-NMT remains elusive.

Metabolic engineering to improve provision of the precursor metabolites L-lysine and $S$-adenosyL-methionine (SAM) may increase de novo production of L-carnitine by the $E$. coli strain constructed here. Strategies to overproduce L-lysine by E. coli are well established (Wendisch, 2020) and an E. coli strain overproducing L-lysine to a titer of $194 \mathrm{~g} \mathrm{~L}^{-1}$ from glucose and ammonium has recently been described (Ye et al., 2020). Regeneration of SAM, the major co-substrate for methyltransferases, using the renewable feedstock methanol as source of the methyl group proved very efficient in E. coli (Okano et al., 2020). Enzyme engineering of E. coli SAM synthetase to reduce product inhibition and increase catalytic activity is a complementary strategy (Wang et al., 2019).

The L-carnitine biosensor fluorescence signal suggested high conversion of L-carnitine from intracellular TML (Figure 5B) as values were comparable to direct L-carnitine supplementation (Kugler et al., 2020). However, only low L-carnitine concentrations were found intra- and extracellularly (Figure 6B), suggesting that the biosensor is sensitive to low intracellular L-carnitine levels and that the export of L-carnitine out of the E. coli cell may also limit biotransformation and its de novo production. Consistent with this hypothesis, transport limitations have also been observed for biotransformation processes converting achiral precursors such as crotonobetaine to L-carnitine using recombinant E. coli strains (Bernal et al., 2007). For biotransformations, this can be overcome by cell permeabilization, for example, with polyethylenimine (Cánovas et al., 2005; Bernal et al., 2007). However, strategies for production of L-carnitine de novo have to maintain the 
cell's integrity. Deletion of the gene for Braun's lipoprotein from the outer membrane of $E$. coli improved L-carnitine production from crotonobetaine without affecting cell growth and metabolism (Bernal et al., 2007; Ni et al., 2007). To prevent re-uptake of L-carnitine secreted from the E. coli cell, the genes coding for L-carnitine uptake systems ProU and ProP were deleted (Verheul et al., 1998; Sevilla et al., 2005a). Engineering L-carnitine export was possible for biotransformation, but is not suitable for de novo production. This is due to the fact that CaiT is an antiporter catalyzing exchange of intracellular and extracellular trimethylammonium compounds such as Lcarnitine, crotonobetaine, and $\gamma$-butyrobetaine, while it does not catalyze uniport of L-carnitine out of the E. coli cell (Jung et al., 2002). A transport system for export of L-carnitine operating in uniport mode is currently not known.

In this work, a proof of concept for the de novo Lcarnitine production in E. coli was shown. The developed fermentation does not depend on petrochemical synthesis of achiral precursors, but instead makes use of renewable feedstocks. Implementation of the L-carnitine biosynthesis pathway was guided by the recently developed L-carnitine biosensor, which allowed the identification of the enzymes and their assembly into an optimized biosynthetic pathway in the form of an enzyme cascade. The biosensor additionally made it possible to identify the precursor supply and product export as current bottlenecks that need to be addressed to further advance L-carnitine de novo production by E. coli. To the best of our knowledge, this is the first description of L-carnitine de novo synthesis using an engineered bacterium.

\section{REFERENCES}

Al Temimi, A. H. K., Pieters, B. J. G. E., Reddy, Y. V., White, P. B., and Mecinović, J. (2016). Substrate scope for trimethyllysine hydroxylase catalysis. Chem. Commun. 52, 12849-12852. doi: 10.1039/C6CC07845A

Arense, P., Bernal, V., Charlier, D., Iborra, J., Foulquié-Moreno, M., and Cánovas, M. (2013). Metabolic engineering for high yielding L(-)-carnitine production in Escherichia coli. Microb. Cell Fact. 12:56. doi: 10.1186/1475-2859-12-56

Bari, M. d. R, Hassan, M., Akai, N., Arima, J., and Mori, N. (2013). Gene cloning and biochemical characterization of 4-N-trimethylaminobutyraldehyde dehydrogenase II from Pseudomonas sp. 13CM. World J. Microbiol. Biotechnol. 29, 683-692. doi: 10.1007/s11274-012-1224-x

Bernal, V., Arense, P., and Cánovas, M. (2016). "L-Carnitine, the vitamin $B_{T}$ : uses and production by the secondary metabolism of Bacteria," in Industrial Biotechnology of Vitamins, Biopigments, and Antioxidants, eds E. J. Vandamme and J. L. Revuelta (Weinheim: Wiley-VCH Verlag GmbH \& Co), 389-419. doi: 10.1002/9783527681754.ch14

Bernal, V., Sevilla, A., Cánovas, M., and Iborra, J. L. (2007). Production of Lcarnitine by secondary metabolism of bacteria. Microb. Cell Fact. 6:31. doi: 10.1186/1475-2859-6-31

Borum, P. R., and Broquist, H. P. (1977). Purification of S-adenosylmethionine: epsilon- $N$-L-lysine methyltransferase. the first enzyme in carnitine biosynthesis. J. Biol. Chem. 252, 5651-5655.

Bracher, S., Hilger, D., Guérin, K., Polyhach, Y., Jeschke, G., Krafczyk, R., et al. (2019). Comparison of the functional properties of trimeric and monomeric CaiT of Escherichia coli. Sci. Rep. 9:3787. doi: 10.1038/s41598-019-40516-7

Cánovas, M., Torroglosa, T., and Iborra, J. L. (2005). Permeabilization of Escherichia coli cells in the biotransformation of trimethylammonium compounds into L-carnitine. Enzyme Microb. Technol. 37, 300-308. doi: 10. 1016/j.enzmictec.2004.07.023

\section{DATA AVAILABILITY STATEMENT}

The original contributions presented in the study are included in the article/Supplementary Material, further inquiries can be directed to the corresponding author/s.

\section{AUTHOR CONTRIBUTIONS}

PK designed the study. PK, MT, and MF conducted the experiments. VW provided funding. MF and VW provided resources. PK, MF, and VW drafted the manuscript. PK and VW finalized the manuscript. All authors read and approved the final version of the manuscript.

\section{FUNDING}

Support for the Article Processing Charge by the Deutsche Forschungsgemeinschaft and the Open Access Publication Fund of Bielefeld University is acknowledged. The funding bodies had no role in the design of the study or the collection, analysis, or interpretation of data or in writing the manuscript.

\section{SUPPLEMENTARY MATERIAL}

The Supplementary Material for this article can be found online at: https://www.frontiersin.org/articles/10.3389/fbioe. 2021.671321/full\#supplementary-material

Castellar, M. R., Cánovas, M., Kleber, H. P., and Iborra, J. L. (1998). Biotransformation of $\mathrm{D}(+)$-carnitine into $\mathrm{L}(-)$-carnitine by resting cells of Escherichia coli O44 K74. J. Appl. Microbiol. 85, 883-890. doi: 10.1046/j.13652672.1998.00601.x

Castellar, M. R., Obón, J. M., Marán, A., Cánovas, M., and Iborra, J. L. (2001). L(-)-carnitine production using a recombinant Escherichia coli strain. Enzyme Microb. Technol. 28, 785-791. doi: 10.1016/s0141-0229(01)0 0332-5

Chen, Y., Abbate, M., Tang, L., Cai, G., Gong, Z., Wei, R., et al. (2014). LCarnitine supplementation for adults with end-stage kidney disease requiring maintenance hemodialysis: a systematic review and meta-analysis. Am. J. Clin. Nutr. 99, 408-422. doi: 10.3945/ajcn.113.062802

Colson, C., Lhoest, J., and Urlings, C. (1979). Genetics of ribosomal protein methylation in Escherichia coli: III. Map position of two genes, prmA and prmB, governing methylation of proteins L11 and L3. Molec. Gen. Genet. 169, 245-250. doi: 10.1007/BF00382270

di Salvo, M. L., Remesh, S. G., Vivoli, M., Ghatge, M. S., Paiardini, A., D’Aguanno, S., et al. (2014). On the catalytic mechanism and stereospecificity of Escherichia coli L-threonine aldolase. FEBS J. 281, 129-145. doi: 10.1111/febs. 12581

DiNicolantonio, J. J., Lavie, C. J., Fares, H., Menezes, A. R., and O’Keefe, J. H. (2013). L-carnitine in the secondary prevention of cardiovascular disease: systematic review and meta-analysis. Mayo Clin. Proc. 88, 544-551. doi: 10. 1016/j.mayocp.2013.02.007

Eichler, K., Bourgis, F., Buchet, A., Kleber, H. P., and Mandrand-Berthelot, M. A. (1994). Molecular characterization of the cai operon necessary for carnitine metabolism in Escherichia coli. Mol. Microbiol. 13, 775-786. doi: 10.1111/j. 1365-2958.1994.tb00470.x

El-Hattab, A. W., and Scaglia, F. (2015). Disorders of carnitine biosynthesis and transport. Mol. Genet. Metab. 116, 107-112. doi: 10.1016/j.ymgme.2015.09.004 
Elssner, T., Engemann, C., Baumgart, K., and Kleber, H.-P. (2001). Involvement of coenzyme A esters and two new enzymes, an Enoyl-CoA hydratase and a CoAtransferase, in the hydration of crotonobetaine to L-carnitine by Escherichia coli. Biochemistry 40, 11140-11148. doi: 10.1021/bi0108812

Fielding, R., Riede, L., Lugo, J., and Bellamine, A. (2018). L-carnitine supplementation in recovery after exercise. Nutrients 10:349. doi: 10.3390/ nu10030349

Flanagan, J. L., Simmons, P. A., Vehige, J., Willcox, M. D., and Garrett, Q. (2010). Role of carnitine in disease. Nutr. Metab. 7:30. doi: 10.1186/1743-7075-7-30

Franken, J., Burger, A., Swiegers, J. H., and Bauer, F. F. (2015). Reconstruction of the carnitine biosynthesis pathway from Neurospora crassa in the yeast Saccharomyces cerevisiae. Appl. Microbiol. Biotechnol. 99, 6377-6389. doi: 10. 1007/s00253-015-6561-x

Funke, M., Diederichs, S., Kensy, F., Müller, C., and Büchs, J. (2009). The baffled microtiter plate: Increased oxygen transfer and improved online monitoring in small scale fermentations. Biotechnol. Bioeng. 103, 1118-1128. doi: 10.1002/bit. 22341

Gibson, D. G., Young, L., Chuang, R.-Y., Venter, J. C., Hutchison, C. A., and Smith, H. O. (2009). Enzymatic assembly of DNA molecules up to several hundred kilobases. Nat. Methods 6, 343-345. doi: 10.1038/nmeth.1318

Gnoni, A., Longo, S., Gnoni, G. V., and Giudetti, A. M. (2020). Carnitine in human muscle bioenergetics: can carnitine supplementation improve physical exercise? Molecules 25:182. doi: 10.3390/molecules25010182

Grand View Research (2019). L-Carnitine Market to Grow at a CAGR of $4.8 \%$ to reach US\$ 262.6 Million from 2019 to 2027. Available online at: https://www. theinsightpartners.com/reports/l-carnitine-marker-market (accessed July 30, 2020).

Green, M. R., Sambrook, J., and Sambrook, J. (2012). Molecular Cloning: A Laboratory Manual. 4th Edition. Cold Spring Harbor, NY: Cold Spring Harbor Laboratory Press.

Gross, C. J., Henderson, L. M., and Savaiano, D. A. (1986). Uptake of L-carnitine, $\mathrm{D}$-carnitine and acetyl-L-carnitine by isolated guinea-pig enterocytes. Biochim. Biophys. Acta, Mol. Cell Res. 886, 425-433. doi: 10.1016/0167-4889(86)90178-3

Gruez, A., Roig-Zamboni, V., Grisel, S., Salomoni, A., Valencia, C., Campanacci, V., et al. (2004). Crystal structure and kinetics identify Escherichia coli YdcW gene product as a medium-chain aldehyde dehydrogenase. J. Mol. Biol. 343, 29-41. doi: 10.1016/j.jmb.2004.08.030

Hassan, M., Okada, M., Ichiyanagi, T., and Mori, N. (2008). 4-NTrimethylaminobutyraldehyde dehydrogenase: purification and characterization of an enzyme from Pseudomonas sp. 13CM. Biosci., Biotechnol., Biochem. 72, 155-162. doi: 10.1271/bbb.70514

Haupka, C. (2020). Chaupka/Codon_Harmonization: Release v1.2.0. Zenodo. Genève, Switzerland: CERN. doi: 10.5281/ZENODO.4062177

Henderson, L. M., Nelson, P. J., and Henderson, L. (1982). Mammalian enzymes of trimethyllysine conversion to trimethylaminobutyrate. Fed. Proc. 41, 28432847.

Horne, D. W., and Broquist, H. P. (1973). Role of lysine and epsilon- $N$ trimethyllysine in carnitine biosynthesis. I. Studies in Neurospora crassa. J. Biol. Chem. 248, 2170-2175.

Hulse, J. D., Ellis, S. R., and Henderson, L. M. (1978). Carnitine biosynthesis. beta-Hydroxylation of trimethyllysine by an alpha-ketoglutarate-dependent mitochondrial dioxygenase. J. Biol. Chem. 253, 1654-1659.

Jung, H., and Kleber, H.-P. (1991). Metabolism of D(+)-carnitine by Escherichia coli. Appl. Microbiol. Biotechnol. 35, 393-395. doi: 10.1007/BF00172731

Jung, H., Buchholz, M., Clausen, J., Nietschke, M., Revermann, A., Schmid, R., et al. (2002). CaiT of Escherichia coli, a new transporter catalyzing L-carnitine $/ \gamma$ butyrobetaine exchange. J. Biol. Chem. 277, 39251-39258. doi: 10.1074/jbc. M206319200

Kabat, M. M., Daniewski, A. R., and Burger, W. (1997). A convenient synthesis of R-(-)-carnitine from R-(-)-epichlorohydrin. Tetrahedron 8, 2663-2665. doi: 10.1016/S0957-4166(97)00324-8

Kang, W.-K., Park, Y.-H., Koh, E.-S., Ju, J.-Y., Lee, J.-H., Choi, H.-J., et al. (2013). A Microorganism of Enterobacteriaceae Genus Haboring Genes Associated With L-Carnitine Biosynthesis and Method of Producing L-Carnitine Using the Microorganism. Available online at: https://patents.google.com/patent/ EP1904620B1/en (accessed September 8, 2020).

Kaufman, R. A., and Broquist, H. P. (1977). Biosynthesis of carnitine in Neurospora crassa. J. Biol. Chem. 252, 7437-7439.
Kensy, F., Zang, E., Faulhammer, C., Tan, R.-K., and Büchs, J. (2009). Validation of a high-throughput fermentation system based on online monitoring of biomass and fluorescence in continuously shaken microtiter plates. Microb. Cell Fact. 8:31. doi: 10.1186/1475-2859-8-31

Klagsbrun, M., and Furano, A. V. (1975). Methylated amino acids in the proteins of bacterial and mammalian cells. Arch. Biochem. Biophys. 169, 529-539. doi: 10.1016/0003-9861(75)90196-4

Kruschwitz, H., McDonald, D., Cossins, E., and Schirch, V. (1993). "Purification of Neurospora crassa cytosolic serine hydroxymethyltransferase," in Chemistry and Biology of Pteridines and Folates Advances in Experimental Medicine and Biology, eds J. E. Ayling, M. G. Nair, and C. M. Baugh (Boston, MA: Springer), 719-722. doi: 10.1007/978-1-4615-2960-6_149

Kugler, P., Fröhlich, D., and Wendisch, V. F. (2020). Development of a biosensor for crotonobetaine-CoA ligase screening based on the elucidation of Escherichia coli carnitine metabolism. ACS Synth. Biol. 9, 2460-2471. doi: 10.1021/ acssynbio.0c00234

Kunjapur, A. M., and Prather, K. L. J. (2015). Microbial engineering for aldehyde synthesis. Appl. Environ. Microbiol. 81, 1892-1901. doi: 10.1128/AEM. 03319-14

Li, J.-M., Li, L.-Y., Zhang, Y.-X., Jiang, Z.-Y., Limbu, S. M., Qiao, F., et al. (2019). Functional differences between L- and D-carnitine in metabolic regulation evaluated using a low-carnitine Nile tilapia model. Br. J. Nutr. 122, 625-638. doi: 10.1017/S000711451900148X

Li, L., Limbu, S. M., Ma, Q., Chen, L., Zhang, M., and Du, Z. (2019). The metabolic regulation of dietary L-carnitine in aquaculture nutrition: present status and future research strategies. Rev. Aquacult. 11, 1228-1257. doi: 10.1111/raq.12289

Lindstedt, G. (1967). Hydroxylation of $\gamma$-butyrobetaine to carnitine in rat liver*. Biochemistry 6, 1271-1282. doi: 10.1021/bi00857a007

Lindstedt, G., and Lindstedt, S. (1970). Cofactor requirements of gammabutyrobetaine hydroxylase from rat liver. J. Biol. Chem. 245, 4178-4186.

Lindstedt, G., Lindstedt, S., and Tofft, M. (1970). Gamma-butyrobetaine hydroxylase from Pseudomonas sp AK 1. Biochemistry 9, 4336-4342. doi: 10. 1021/bi00824a014

Lindstedt, G., Lindstedt, S., Midtvedt, T., and Tofft, M. (1967). The Formation and degradation of carnitine in Pseudomonas ${ }^{*}$ Biochemistry 6, 1262-1270. doi: 10.1021/bi00857a006

Longo, N., Frigeni, M., and Pasquali, M. (2016). Carnitine transport and fatty acid oxidation. Biochim. Biophys. Acta, Mol. Cell Res. 1863, 2422-2435. doi: 10.1016/j.bbamcr.2016.01.023

Magoulas, P. L., and El-Hattab, A. W. (2012). Systemic primary carnitine deficiency: an overview of clinical manifestations, diagnosis, and management. Orphanet J. Rare. Dis. 7:68. doi: 10.1186/1750-1172-7-68

Meyer, H.-P., and Robins, K. T. (2005). Large scale bioprocess for the production of optically pure L-carnitine. Monatsh. Chem. 136, 1269-1277. doi: 10.1007/ s00706-005-0330-y

Naidu, G. S. N., Lee, I. Y., Lee, E. G., Kang, G. H., and Park, Y. H. (2000). Microbial and enzymatic production of L-carnitine. Bioprocess Eng. 23, 627-635. doi: $10.1007 / \mathrm{s} 004490000212$

Nakamura, Y. (2000). Codon usage tabulated from international DNA sequence databases: status for the year 2000. Nucleic Acids Res. 28, 292-292. doi: 10.1093/ nar/28.1.292

Ni, Y., Reye, J., and Chen, R. R. (2007). lpp deletion as a permeabilization method. Biotechnol. Bioeng. 97, 1347-1356. doi: 10.1002/bit.21375

Obón, J. M., Maiquez, J. R., Cánovas, M., Kleber, H.-P., and Iborra, J. L. (1999). High-density Escherichia coli cultures for continuous L(-)carnitine production. Appl. Microbiol. Biotechnol. 51, 760-764. doi: 10.1007/ s002530051459

Okano, K., Sato, Y., Inoue, S., Kawakami, S., Kitani, S., and Honda, K. (2020). Enhancement of $S$-adenosylmethionine-dependent methylation by integrating methanol metabolism with 5-Methyl-tetrahydrofolate formation in Escherichia coli. Catalysts 10:1001. doi: 10.3390/catal100 91001

Parvanova, A., Trillini, M., Podestà, M. A., Iliev, I. P., Aparicio, C., Perna, A., et al. (2018). Blood Pressure and metabolic effects of Acetyl-L-carnitine in Type 2 diabetes: DIABASI randomized controlled trial. J. Endocr. Soc. 2, 420-436. doi: 10.1210/js.2017-00426

Preusser, A., Wagner, U., Elssner, T., and Kleber, H.-P. (1999). Crotonobetaine reductase from Escherichia coli consists of two proteins. Biochim. Biophys. 
Acta, Protein Struct. Mol. Enzymol. 1431, 166-178. doi: 10.1016/S0167-4838(99) 00032- 1

Rebouche, C. J. (1977). Carnitine movement across muscle cell membranes. Studies in isolated rat muscle. Biochim. Biophys. Acta, Biomembr. 471, 145-155. doi: 10.1016/0005-2736(77)90402-3

Rebouche, C. J. (1983). Effect of dietary carnitine isomers and $\gamma$-butyrobetaine on L-carnitine biosynthesis and metabolism in the rat. J. Nutr. 113, 1906-1913. doi: $10.1093 / \mathrm{jn} / 113.10 .1906$

Reddy, Y. V., Al Temimi, A. H. K., White, P. B., and Mecinoviæ, J. (2017). Evidence that trimethyllysine hydroxylase catalyzes the formation of $(2 S, 3 S)$ 3-hydroxy- $N^{\varepsilon}$-trimethyllysine. Org. Lett. 19, 400-403. doi: 10.1021/acs.orglett. $6 \mathrm{~b} 03608$

Rehman, Z., Naz, S., Khan, R. U., and Tahir, M. (2017). An update on potential applications of L-carnitine in poultry. World's Poult. Sci. J. 73, 823-830. doi: $10.1017 /$ S0043933917000733

Reuter, S. E., and Evans, A. M. (2012). Carnitine and acylcarnitines: pharmacokinetic, pharmacological and clinical aspects. Clin. Pharmacokinet. 51, 553-572. doi: 10.1007/BF03261931

Ringseis, R., Keller, J., and Eder, K. (2018a). Basic mechanisms of the regulation of L-carnitine status in monogastrics and efficacy of L-carnitine as a feed additive in pigs and poultry. J. Anim. Physiol. Anim. Nutr. 102, 1686-1719. doi: $10.1111 /$ jpn. 12959

Ringseis, R., Keller, J., and Eder, K. (2018b). Regulation of carnitine status in ruminants and efficacy of carnitine supplementation on performance and health aspects of ruminant livestock: a review. Arch. Anim. Nutr. 72, 1-30. doi: 10.1080/1745039X.2017.1421340

Sachan, D. S., and Hoppel, C. L. (1980). Carnitine biosynthesis. Hydroxylation of N6-trimethyl-lysine to 3-hydroxy-N6-trimethyl-lysine. Biochem. J. 188, 529534. doi: 10.1042/bj1880529

Samsonova, N. N., Smirnov, S. V., Novikova, A. E., and Ptitsyn, L. R. (2005). Identification of Escherichia coli $\mathrm{K} 12 \mathrm{YdcW}$ protein as a $\gamma$-aminobutyraldehyde dehydrogenase. FEBS Lett. 579, 4107-4112. doi: 10.1016/j.febslet.2005.06.038

Schirch, V., Hopkins, S., Villar, E., and Angelaccio, S. (1985). Serine hydroxymethyltransferase from Escherichia coli: purification and properties. J. Bacteriol. 163, 1-7. doi: 10.1128/JB.163.1.1-7.1985

Schneider, B. L., and Reitzer, L. (2012). Pathway and enzyme redundancy in putrescine catabolism in Escherichia coli. J. Bacteriol. 194, 4080-4088. doi: 10.1128/JB.05063-11

Seim, H., Ezold, R., Kleber, H.-P., Strack, E., and Seim, H. (1980). Stoffwechsel des L-Carnitins bei Enterobakterien. Zeitschr. Allgem. Mikrobiol. 20, 591-594.

Sevilla, A., Schmid, J. W., Mauch, K., Iborra, J. L., Reuss, M., and Cánovas, M. (2005a). Model of central and trimethylammonium metabolism for optimizing L-carnitine production by E. coli. Metab. Eng. 7, 401-425. doi: 10.1016/j.ymben. 2005.06.005

Sevilla, A., Vera, J., Díaz, Z., Cánovas, M., Torres, N. V., and Iborra, J. L. (2005b). Design of metabolic engineering strategies for maximizing L-()-carnitine production by Escherichia coli. Integration of the metabolic and bioreactor levels. Biotechnol. Prog. 21, 329-337. doi: 10.1021/bp049 7583

Song, X., Qu, H., Yang, Z., Rong, J., Cai, W., and Zhou, H. (2017). Efficacy and safety of L-carnitine treatment for chronic heart failure: a meta-analysis of randomized controlled trials. BioMed Res. Int. 2017, 1-11. doi: 10.1155/2017/ 6274854

Sophos, N. A., and Vasiliou, V. (2003). Aldehyde dehydrogenase gene superfamily: the 2002 update. Chem. Biol. Interact. 14, 5-22. doi: 10.1016/S0009-2797(02) 00163- 1

Strijbis, K., Van Roermund, C. W. T., Hardy, G. P., Van den Burg, J., Bloem, K., Haan, J., et al. (2009). Identification and characterization of a complete carnitine biosynthesis pathway in Candida albicans. FASEB J. 23, 2349-2359. doi: 10.1096/fj.08-127985
Strijbis, K., Vaz, F. M., and Distel, B. (2010). Enzymology of the carnitine biosynthesis pathway. IUBMB Life 62, 357-362. doi: 10.1002/iub.323

Swiegers, J. H., Vaz, F. M., Pretorius, I. S., Wanders, R. J. A., and Bauer, F. F. (2002). Carnitine biosynthesis in Neurospora crassa: identification of a cDNA coding for $\varepsilon$-N-trimethyllysine hydroxylase and its functional expression in Saccharomyces cerevisiae. FEMS Microbiol. Lett. 210, 19-23. doi: 10.1111/j.1574-6968.2002. tb11154.x

The Insight Partners (2020). L-Carnitine Market Size, Share, Trends | Global Industry Report, 2025. Available online at: https://www.grandviewresearch. com/industry-analysis/l-carnitine-market (accessed July 30, 2020).

Trievel, R. C., Flynn, E. M., Houtz, R. L., and Hurley, J. H. (2003). Mechanism of multiple lysine methylation by the SET domain enzyme Rubisco LSMT. Nat. Struct. Mol. Biol. 10, 545-552. doi: 10.1038/nsb946

Vanet, A., Plumbridge, J. A., Guérin, M.-F., and Alix, J.-H. (1994). Ribosomal protein methylation in Escherichia coli: the gene prmA, encoding the ribosomal protein L11 methyltransferase, is dispensable. Mol. Microbiol. 14, 947-958. doi: 10.1111/j.1365-2958.1994.tb01330.x

Vaz, F. M., and Wanders, R. J. A. (2002). Carnitine biosynthesis in mammals. Biochem. J. 361, 417-429. doi: 10.1042/bj3610417

Vaz, F. M., Ofman, R., Westinga, K., Back, J. W., and Wanders, R. J. A. (2001). Molecular and Biochemical characterization of Rat $\varepsilon-N$-trimethyllysine hydroxylase, the first enzyme of carnitine biosynthesis. J. Biol. Chem. 276, 33512-33517. doi: 10.1074/jbc.M105929200

Verheul, A., Wouters, J. A., Rombouts, F. M., and Abee, T. (1998). A possible role of ProP, ProU and CaiT in osmoprotection of Escherichia coli by carnitine. J. Appl. Microbiol. 85, 1036-1046. doi: 10.1111/j.1365-2672.1998.tb05269.x

Veronese, N., Stubbs, B., Solmi, M., Ajnakina, O., Carvalho, A. F., and Maggi, S. (2018). Acetyl-L-carnitine supplementation and the treatment of depressive symptoms: a systematic review and meta-analysis. Psychosom. Med. 80, 154159. doi: 10.1097/PSY.0000000000000537

Volkmer, B., and Heinemann, M. (2011). Condition-dependent cell volume and concentration of Escherichia coli to facilitate data conversion for systems biology modeling. PLoS One 6:e23126. doi: 10.1371/journal.pone.0023126

Wang, X., Jiang, Y., Wu, M., Zhu, L., Yang, L., and Lin, J. (2019). Semi-rationally engineered variants of $S$-adenosylmethionine synthetase from Escherichia coli with reduced product inhibition and improved catalytic activity. Enzyme Microb. Technol. 129:109355. doi: 10.1016/j.enzmictec.2019.05.012

Wargo, M. J., and Meadows, J. A. (2015). Carnitine in bacterial physiology and metabolism. Microbiology 161, 1161-1174. doi: 10.1099/mic.0.000080

Wendisch, V. F. (2020). Metabolic engineering advances and prospects for amino acid production. Metab. Eng. 58, 17-34. doi: 10.1016/j.ymben.2019.03.008

Ye, C., Luo, Q., Guo, L., Gao, C., Xu, N., Zhang, L., et al. (2020). Improving lysine production through construction of an Escherichia coli enzymeconstrained model. Biotechnol. Bioeng. 117, 3533-3544. doi: 10.1002/bit.2 7485

Zhang, M., Xu, J.-Y., Hu, H., Ye, B.-C., and Tan, M. (2018). Systematic proteomic analysis of protein methylation in prokaryotes and eukaryotes revealed distinct substrate specificity. Proteomics 18:1700300. doi: 10.1002/pmic.2017 00300

Conflict of Interest: The authors declare that the research was conducted in the absence of any commercial or financial relationships that could be construed as a potential conflict of interest.

Copyright (C) 2021 Kugler, Trumm, Frese and Wendisch. This is an open-access article distributed under the terms of the Creative Commons Attribution License (CC BY). The use, distribution or reproduction in other forums is permitted, provided the original author(s) and the copyright owner(s) are credited and that the original publication in this journal is cited, in accordance with accepted academic practice. No use, distribution or reproduction is permitted which does not comply with these terms. 